\title{
Molecular and cell-biological mechanisms of antigen cross-presentation
}

\section{Christian Kurts*}

Institute of Molecular Medicine and Experimental Immunology, Friedrich Wilhelms-Universität Bonn, Bonn, Germany

${ }^{*}$ Correspondence: ckurts@uni-bonn.de

Edited by:

Hermann Wagner, Technical University Munich, Germany

Reviewed by:

Hermann Wagner, Technical University Munich, Germany

Cross-priming serves to activate cytotoxic $\mathrm{T}$ lymphocytes for immune defense against viruses and tumors and plays an important role in vaccinations. Only certain dendritic cell (DC) subsets can cross-present. Several cell surface markers have been described that more or less specifically and sensitively characterize these subsets. The cell-biological mechanism(s) why a DC subset can cross-present are less clear. Theoretically, the task of cross-presentation can be divided into several mechanistic steps: (1) Antigen uptake by various endocytosis mechanisms, (2) Intracellular antigen routing into distinct organelles including the crossing of organelle membranes, (3) Antigen processing into peptides, (4) Peptide loading onto MHC molecules, and (5) Transport of these complexes to the cell surfaces for presentation to T cells. Each of these steps is dependent on numerous parameters, not only the DC subtype, but also the nature of the antigen or

\section{REFERENCES}

Chopin, M., Allan, R. S., and Belz, G. T. (2012). Transcriptional regulation of dendritic cell diversity. Front. Immunol. 3:26. doi: 10.3389/fimmu.2012.00026

Compeer, E. B., Flinsenberg, T. W. H., van der Grein, S. G., and Boes, M. (2012). Antigen processing and remodeling of the endosomal pathway: requirements for antigen crosspresentation. Front. Immunol. 3:37. doi: 10.3389/fimmu.2012.00037

Harriff, M. J., Purdy, G. E., and Lewinsohn, D. M. (2012). Escape from the phagosome: the explanation for MHC-I processing of mycobacterial antigens? Front. Immunol. 3:40. doi: 10.3389/fimmu.2012.00040
Kreer, C., Rauen, J., Zehner, M., and Burgdorf, S. (2012). Cross-presentation: how to get there - or how to get the ER. Front. Immunol. 2:87. doi: 10.3389/fimmu.2011.00087

Kroczek, R. A., and Henn, V. (2012). The role of XCR1 and its ligand XCL1 in antigen cross-presentation by murine and human dendritic cells. Front. Immunol. 3:14. doi: 10.3389/fimmu.2012.00014

Murshid, A., Gong, J., and Calderwood, S. K. (2012). The role of heat shock proteins in antigen cross presentation. Front. Immunol. 3:63. doi: 10.3389/fimmu.2012.00063

Neefjes, J., and Sadaka, C. (2012). Into the intracellular logistics of cross-presentation. Front.

the presence of further signals that impact DC function or signify the presence of danger or infection.

This research topic contains 10 articles by leading experts in the field of antigen presentation that cover our current knowledge on the molecular mechanisms underlying cross-presentation (Chopin et al., 2012; Compeer et al., 2012; Harriff et al., 2012; Kreer et al., 2012; Kroczek and Henn, 2012; Murshid et al., 2012; Neefjes and Sadaka, 2012; Saveanu and van Endert, 2012; Thacker and Janssen, 2012; Wagner et al., 2012). The authors describe the influence of endocytosis receptors or heat shock proteins for antigen uptake, the intracellular logistics of antigen routing, membrane translocation mechanisms and proteases, the transcriptional DC regulation, the chemokine-mediated crosstalk between cross-presenting DCs and the cytotoxic $\mathrm{T}$ cells to be cross-primed and immune-escape mechanisms of pathogens.

Immunol. 3:31. doi: 10.3389/fimmu. 2012.00031

Saveanu, L., and van Endert, P. (2012). The role of insulin-regulated aminopeptidase in MHC class I antigen presentation. Front. Immunol. 3:57. doi: 10.3389/fimmu. 2012.00057

Thacker, R. I., and Janssen, E. M. (2012). Cross-presentation of cell-associated antigens by mouse splenic dendritic cell populations. Front. Immunol. 3:41. doi: 10.3389/ fimmu.2012.00041

Wagner, C. S., Grotzke, J. E., and Cresswell, P. (2012). Intracellular events regulating cross-presentation. Front. Immunol. 3:138. doi: 10.3389/fimmu. 2012.00138
Received: 01 February 2013; accepted: 10 February 2013; published online: 28 February 2013.

Citation: Kurts C (2013) Molecular and cell-biological mechanisms of antigen cross-presentation. Front. Immunol. 4:51. doi: 10.3389/fimmu.2013.00051

This article was submitted to Frontiers in Antigen Presenting Cell Biology, a specialty of Frontiers in Immunology. Copyright (C) 2013 Kurts. This is an open-access article distributed under the terms of the Creative Commons Attribution License, which permits use, distribution and reproduction in other forums, provided the original authors and source are credited and subject to any copyright notices concerning any third-party graphics etc. 